\title{
CPPU may induce gibberellin-independent parthenocarpy associated with PbRR9 in 'Dangshansu' pear
}

Liu Cong ${ }^{1}$, Ting Wu', Hanting Liu', Huibin Wang ${ }^{1}$, Haiqi Zhang ${ }^{1}$, Guangping Zhao ${ }^{1}$, Yao Wen', Qianrong Shi', Lingfei $\mathrm{Xu}^{1}$ and Zhigang Wang ${ }^{1}$

\begin{abstract}
Parthenocarpy is a valuable trait in self-incompatible plants, such as pear. N-(2-chloro-4-pyridyl)- $N^{\prime}$-phenylurea (CPPU), a synthetic cytokinin analog, can induce parthenocarpy in pear (Pyrus spp.), but the mechanism of induction is unclear. To investigate the role of gibberellin in CPPU-induced parthenocarpy in pear, CPPU supplemented with paclobutrazol (PAC) was sprayed onto 'Dangshansu' pear. We found that the fruit set rate of pear treated with CPPU supplemented with PAC was identical to that in a CPPU-alone treatment group. In regard to cell development, CPPU mainly promoted hypanthium cell division and expansion, and PAC application had no influence on CPPU-induced cell development. RNA sequencing revealed that gibberellin 20 oxidase and gibberellin 3 oxidase genes were not differentially expressed following CPPU treatment. According to the analysis of fruit phytohormone content, the CPPU treatments did not induce gibberellin biosynthesis. These results suggest that CPPU-induced parthenocarpy may be gibberellin independent in 'Dangshansu' pear. After CPPU treatment, the indole acetic acid (IAA) content in fruit was significantly increased, and the abscisic acid (ABA) content was significantly decreased. Similarly, RNA sequencing revealed that many genes involved in the auxin and ABA pathways were significantly differentially expressed in the CPPU treatment groups; among them, indole-3-pyruvate monooxygenase (YUCCA) was significantly upregulated and 9-cis-epoxycarotenoid dioxygenase (NCED) was significantly downregulated. IAA and ABA may thus play important roles in CPPU-induced parthenocarpy. PbTwo-component response regulator9 (PbRR9), PbYUCCA4, and PbNCED6 were then selected to further elucidate the mechanism of CPPU-induced parthenocarpy. A yeast one-hybrid assay indicated that PbRR9 can combine with the PbYUCCA4 and PbNCED6 promoters. Dual luciferase assays revealed that PbRR9 can promote and repress the activities of the PbYUCCA4 and PbNCED6 promoters, respectively. After the transient expression of PbRR9 in fruits, PbYUCCA4 expression was significantly upregulated, and PbNCED6 expression was significantly downregulated. This study uncovered a CPPU-induced parthenocarpy mechanism that is different from that in tomato. CPPU may upregulate PbYUCCA4 and downregulate PbNCED6 by upregulating PbRR9, thereby increasing IAA content and decreasing ABA content to ultimately induce parthenocarpy in 'Dangshansu' pear. However, because only a single time point was used and because 'botanical' and 'accessory' fruits have different structures, this conclusion is still preliminary.
\end{abstract}

Correspondence: Lingfei Xu (lingfxu2013@sina.com) or

Zhigang Wang (wzhg001@163.com)

${ }^{1}$ College of Horticulture, Northwest A\&F University, Taicheng, Road No.3,

Yangling, Shaanxi, Province, China

\section{Introduction}

Fruit set, which generally requires successful pollination and fertilization, initiates fruit development ${ }^{1,2}$. Pollination and subsequent fertilization are sensitive to environmental conditions ${ }^{3}$, such as low temperatures. Parthenocarpy is the phenomenon in which an unfertilized ovary

\section{(c) The Author(s) 2020}

(c) (i) Open Access This article is licensed under a Creative Commons Attribution 4.0 International License, which permits use, sharing, adaptation, distribution and reproduction c. in any medium or format, as long as you give appropriate credit to the original author(s) and the source, provide a link to the Creative Commons license, and indicate if changes were made. The images or other third party material in this article are included in the article's Creative Commons license, unless indicated otherwise in a credit line to the material. If material is not included in the article's Creative Commons license and your intended use is not permitted by statutory regulation or exceeds the permitted use, you will need to obtain permission directly from the copyright holder. To view a copy of this license, visit http://creativecommons.org/licenses/by/4.0/. 
develops into a seedless fruit ${ }^{4}$. Pear is naturally selfincompatible and requires commercial producers to plant pollinizer trees to achieve adequate fertilization and fruit set. Understanding the mechanisms of parthenocarpy is beneficial, as it offers the potential to reduce production costs and increase fruit yield.

Parthenocarpy is closely related to the coordinated action of different hormones produced in unpollinated ovaries $^{5}$. Following successful pollination and fertilization, auxin synthesis is activated in the ovary, and the endogenous auxin content significantly increases to a level sufficient to induce fruit development ${ }^{6}$. Auxin was the first phytohormone discovered to induce parthenocarpy in plants ${ }^{7,8}$. Previous studies have indicated that an ectopic supply of auxin or auxin-like substances can induce parthenocarpy in strawberry ${ }^{9}$, tomato ${ }^{10}$, pear ${ }^{11,12}$, and eggplant ${ }^{13}$. In addition, changes in auxin biosynthesis and signaling pathways have been shown to induce parthenocarpic fruit set and fruit development. In tobacco and eggplant, the overexpression of the ovulespecific auxin biosynthesis gene DefH9::tryptophan 2monooxygenase (iaaM) results in parthenocarpy ${ }^{14}$. When overexpressed in tomato, the auxin receptor transport inhibitor response (TIR) is responsible for parthenocarpic fruit $^{15}$. The downregulation of the negative regulator auxin response factor 7 (ARF7) in tomato leads to parthenocarpic fruit development, thus indicating that the reduced expression of indole acetic acid (IAA) repressor genes can result in parthenocarpy ${ }^{16}$. Similarly, indoleacetic acid-induced protein 9 (SIIAA9)-downregulated transgenic tomato lines also present a parthenocarpic phenotype $^{17,18}$. Gibberellin is another important hormone that contributes to parthenocarpy. The exogenous application of gibberellins can induce fertilizationindependent fruit development in apple ${ }^{19}, \operatorname{rose}^{20}$, loquat $^{21}$, grape ${ }^{22}$, and pear ${ }^{23,24}$. The gibberellin content in plants is regulated by the expression of gibberellin oxidase (GAox) ${ }^{25}$, whose influence has also been implicated in the occurrence of parthenocarpy. In tomato, overexpression of gibberellin 20-oxidase and silencing of gibberellin 2-oxidase can both lead to the production of parthenocarpic fruits in the absence of pollination ${ }^{26,27}$. Similar to auxin-related parthenocarpy, RNAi silencing of DELLA - the key repressor of the gibberellin signaling pathway-can induce parthenocarpic fruit development $^{28}$. Crosstalk between auxin and gibberellin has been reported in tomato and pear, with auxin being upstream of gibberellin in parthenocarpy ${ }^{10,12}$.

In addition to auxin and gibberellin, cytokinin induces parthenocarpy in horticultural plants ${ }^{29-32}$, but the mechanism of cytokinin-induced parthenocarpy is not understood. Although $N$-(2-chloro-4-pyridyl)- $N$ '-phenylurea (CPPU), a synthetic cytokinin analog, induces parthenocarpic fruit development in pear $^{23}$, the mechanism of CPPU-induced parthenocarpy in this species has not been clearly elucidated. In this study, we sprayed 'Dangshansu' pear with CPPU to induce parthenocarpy and then investigated the underlying mechanism.

\section{Results \\ CPPU may induce GA-independent parthenocarpy in 'Dangshansu' pear}

In the hand-pollinated group, the fruit set rate reached $83.8 \%$ (Table 1). The fruit set rate of the CPPU treatment group was $93.5 \%$, which was significantly higher than that of the pollinated group (Table 1). Because fruit set is an important and fundamental process in parthenocarpy, these results suggest that CPPU may induce parthenocarpy in 'Dangshansu' pear. To investigate the role of gibberellin (GA) in CPPU-induced parthenocarpy, we treated flowers with CPPU supplemented with PAC, a GA biosynthesis inhibitor. We found that PAC-supplemented CPPU still led to parthenocarpy in 'Dangshansu' pear; the fruit set rate reached $94.3 \%$, which was not significantly different from that in the CPPU-alone treatment group (Table 1). We thus speculated that CPPU induces parthenocarpy in 'Dangshansu' pear that may be independent of GA.

\section{Morphological and histological observations of CPPU and CPPU + PAC-induced parthenocarpic fruits}

The fruits in each treatment group exhibited morphological differences at 4 DAA. Pollinated fruits and fruits treated with either CPPU alone or PAC-supplemented CPPU appeared to be larger than unpollinated fruits (Fig. 1). Fruits from pollinated, CPPU-alone, and PACsupplemented CPPU treatment groups were also larger than unpollinated fruits at 10 DAA (Fig. 1). Both CPPUalone and PAC-supplemented CPPU treatments induced fleshy calyxes at 10 DAA, which led to the formation of fruits that were more deeply lobed (Fig. 1). No mature fruits were produced in the unpollinated group, whereas mature fruits were obtained at 152 DAA from pollinated, CPPU-alone, and PAC-supplemented CPPU treatments. The latter two treatments generated seedless fruits

\section{Table 1 The effects of $N$-(2-chloro-4-pyridyl)- $N^{\prime}-$ phenylurea (CPPU) and paclobutrazol (PAC) on 'Dangshansu' pear fruit set}

\begin{tabular}{lllll}
\hline Treatment & UP & P & CPPU & CPPU + PAC \\
\hline Fruit set rate (\%) & Oc & $83.8 \pm 2.58 \mathrm{~b}$ & $93.5 \pm 1.56 \mathrm{a}$ & $94.3 \pm 1.28 \mathrm{a}$ \\
\hline
\end{tabular}

UP unpollinated, $P$ pollinated, $C P P U$ treatment with $C P P U$ alone, $C P P U+P A C$ treatment with PAC-supplemented CPPU. Significant differences among treatments as determined by one-way ANOVA $(P<0.05)$ are indicated using different lowercase letters $(a, b, c)$. Error bars represent the standard deviation of the mean (SD; $n=3$ ) 


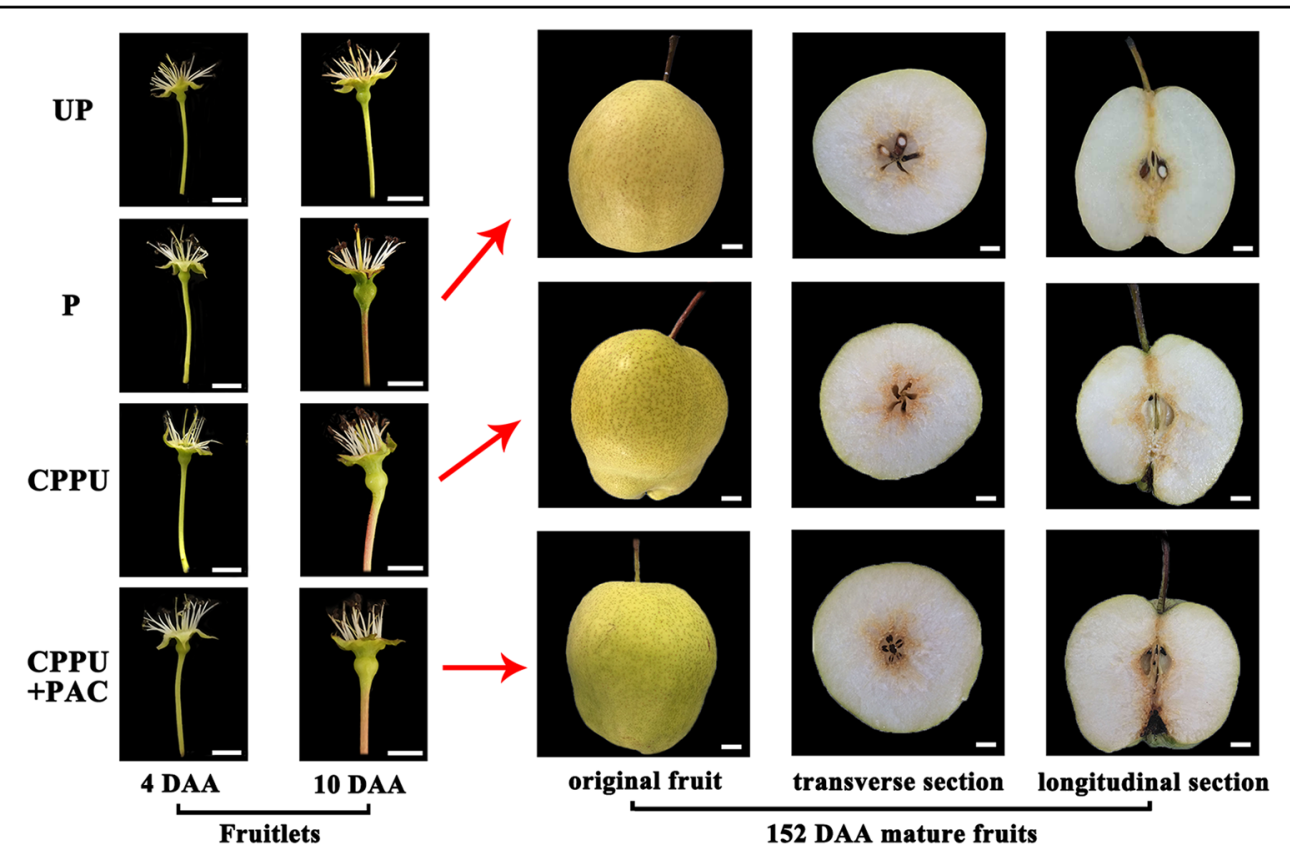

Fig. 1 Morphology of 'Dangshansu' pear fruits. UP, unpollinated; P, pollinated; CPPU, treatment with N-(2-chloro-4-pyridyl)- $N^{\prime}$-phenylurea alone; CPPU+PAC, treatment with N-(2-chloro-4-pyridyl)- $N^{\prime}$-phenylurea supplemented with paclobutrazol. Fruits were collected at 4, 10, and 152 days after anthesis (DAA). Bar $=1 \mathrm{~cm}$

(Supplementary Fig. S1). No significant difference was observed in the weights of pollinated, CPPU-treated, and PAC-supplemented CPPU-treated fruits (Fig. 1; Supplementary Table S1), and parthenocarpic fruits induced by CPPU-alone and PAC-supplemented CPPU treatments were less firm than control fruits (Supplementary Table S1). Moreover, the core transverse diameter of mature seedless fruits was significantly smaller than that of normal fruits (Supplementary Table S1).

To further observe the effects of CPPU treatment on cell development, paraffin slices were prepared from fruits collected at 4 DAA. We found that the pericarp and hypanthium of pollinated fruits were significantly thicker than those of fruits in the unpollinated group, while the CPPU-alone and PAC-supplemented CPPU treatments mainly promoted hypanthium development (Fig. 2a-e, h). Pollination significantly increased the pericarp and hypanthium cell areas (Fig. 2f, i). After treatment with CPPU alone or CPPU supplemented with PAC, the hypanthium cell areas and cell layers were significantly larger than those in the unpollinated treatment (Fig. 2i, j). No significant differences in pericarp and hypanthium thickness, cell area, or the number of cell layers were detected between the CPPU-alone and PACsupplemented CPPU treatment groups (Fig. 2e-j). These results suggest that CPPU increases hypanthium thickness by promoting cell division and cell expansion at the early fruit development stage and that this process may be independent of gibberellin.

\section{Phytohormone levels in CPPU-induced parthenocarpic fruits}

Because phytohormone application was found to play a key role in promoting parthenocarpy, we measured fruit phytohormone levels. $\mathrm{GA}_{3}$ and $\mathrm{GA}_{4}$ are the two major active gibberellins in pear. No differences were observed in the $\mathrm{GA}_{3}$ contents of fruits from the CPPU-alone and PAC-supplemented CPPU treatment groups compared with that in the unpollinated group at 4 DAA (Fig. 3a). In contrast, the $\mathrm{GA}_{4}$ contents of fruits decreased significantly in the CPPU-alone and PAC-supplemented CPPU treatment groups (Fig. 3b). A significant increase in IAA content was detected in the CPPU-alone and PACsupplemented CPPU treatment groups compared with that in the unpollinated group at 4 DAA, with the IAA content increased significantly, by five-fold, after CPPU treatment (Fig. 3c). In addition, the ABA content of fruits in the CPPU-alone and PAC-supplemented CPPU treatment groups was significantly decreased at 4 DAA (Fig. 3d). These results support our speculation that CPPUinduced parthenocarpy is independent of GA in 'Dangshansu' pear. Moreover, IAA and ABA may play important roles in this process.

\section{Transcriptome analysis of CPPU-induced parthenocarpic fruits}

CPPU took effect rapidly, as cell development and fruit hormone contents among treatment groups exhibited differences at 4 DAA. To investigate the mechanism of 


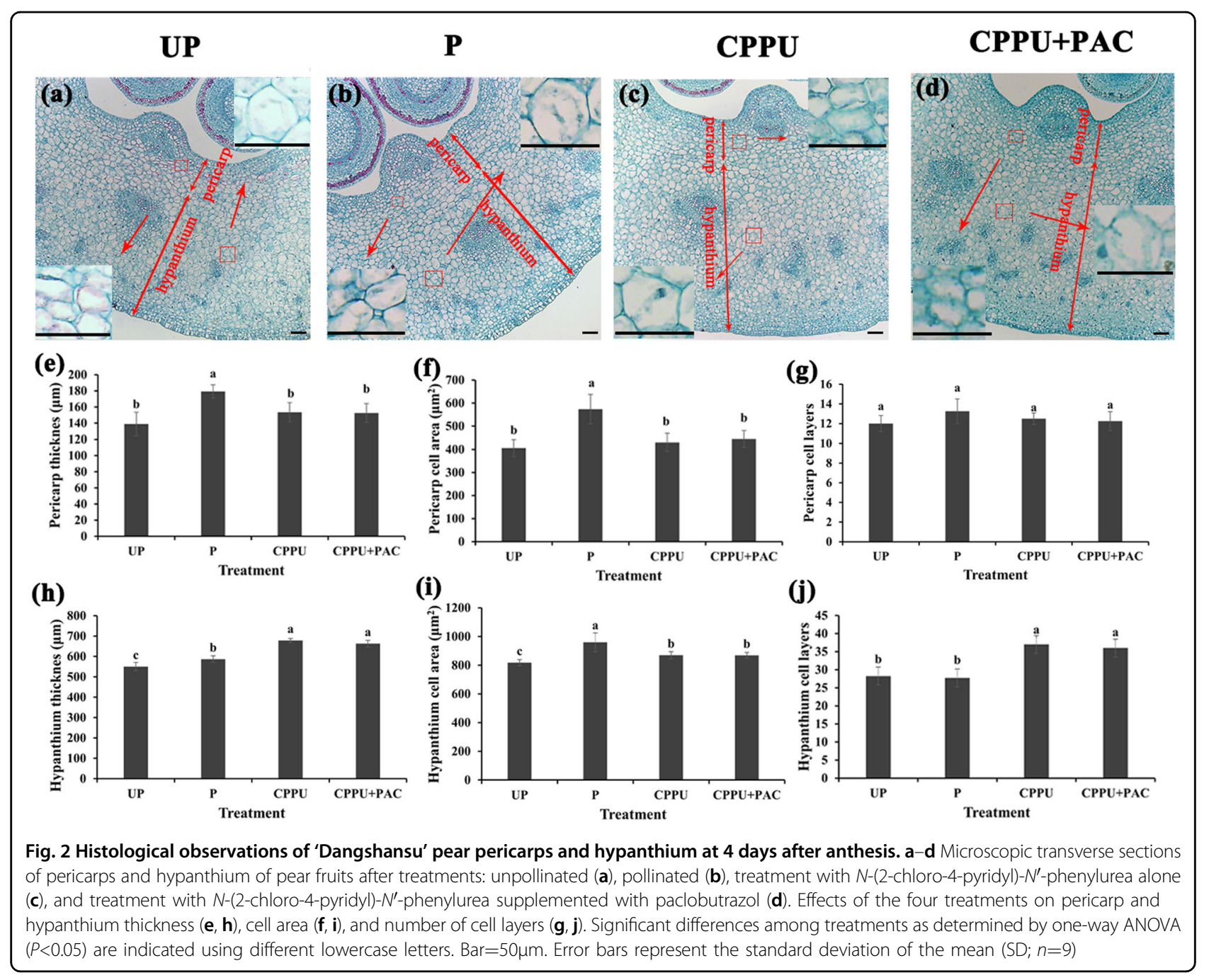

CPPU-induced parthenocarpy, we therefore selected fruits collected at 4 DAA for transcriptome analysis. As shown in Supplementary Table S2, 125-bp/150-bp pairedend reads mapped to the pear genome at an average rate of $78 \%^{33}$. After mapping sequence reads to the pear genome, we performed a differential expression analysis. This analysis identified 3969, 4954, and 7422 differentially expressed genes (DEGs) present in the pollinated, CPPUalone, and PAC-supplemented CPPU treatment groups, respectively, compared with the unpollinated group (Fig. 4a). A total of 995 genes were differentially expressed in all three groups (Fig. 4a). To verify the RNAseq results, seven genes that were relevant and/or interesting were selected for qRT-PCR analysis (Supplementary Fig. S2).

\section{DEGs involved in cell expansion and cell division}

On the basis of known functions, 48 and 54 cell development-related DEGs were detected in the CPPUalone and PAC-supplemented CPPU treatment groups, respectively (Fig. 4b; Supplementary Table S3). After treatment with CPPU alone or PAC-supplemented CPPU, 28 and 33 cyclin genes, respectively, were significantly upregulated (Fig. 4b; Supplementary Table S3); among them, 12 were upregulated more than twofold in both CPPU treatment groups (Fig. 4b; Supplementary Table S3). Genes related to expansins were also upregulated. Three expansin genes-Pbexpansin 2 (LOC103940640), Pbexpansin1 (LOC103953799), and Pbexpansin-A8 (LOC103953745)-were upregulated nearly four-fold in both the CPPU-alone and PACsupplemented CPPU treatment groups (Fig. 4b; Supplementary Table S3). Xyloglucan endo-transglucosylases (XTHs) catalyze xyloglucan endohydrolysis and endotransglycosylation, which are processes related to cell expansion. The analysis of the RNA sequencing data revealed that five and four $X T H s$ were upregulated more than two-fold after the CPPU-alone and PACsupplemented CPPU treatments, respectively (Fig. 4b; Supplementary Table S3). PbXTH32 (LOC103965682) was upregulated eight-fold in both the CPPU-alone and 
(a)

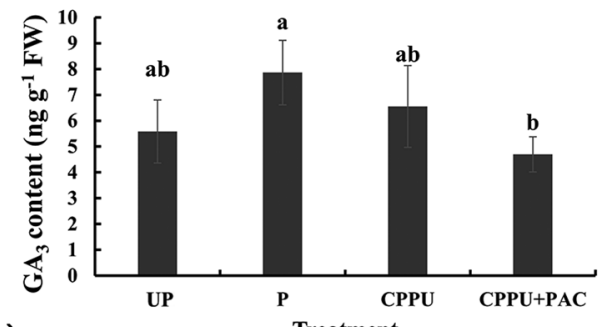

(c)

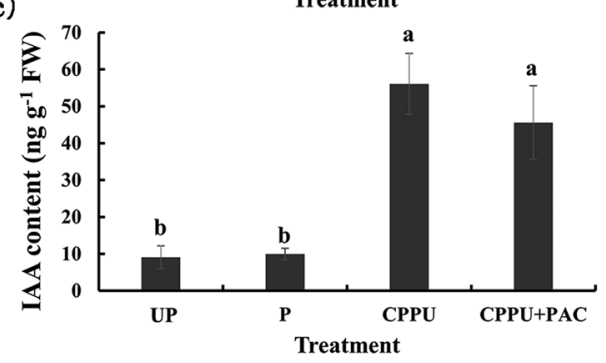

(b)

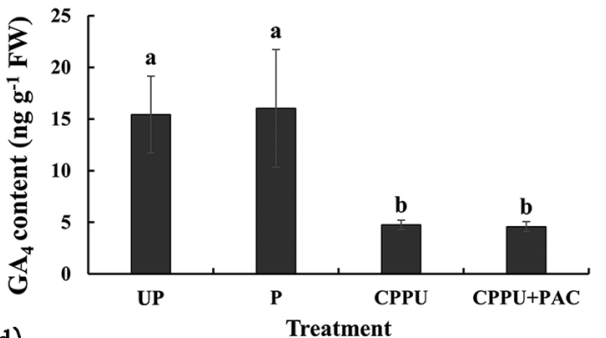

(d)

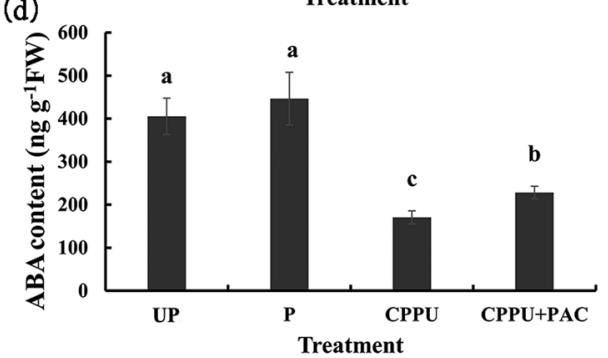

Fig. 3 Phytohormone levels in 'Dangshansu' pear fruits. Phytohormone levels in 'Dangshansu' pear fruits. a-c Concentrations of the plant phytohormones $\mathrm{GA}_{3}(\mathbf{a}), \mathrm{GA}_{4}(\mathbf{b}), \mathrm{IAA}(\mathbf{c})$, and $\mathrm{ABA}(\mathbf{d})$ in fruits collected at 4 days after anthesis. Phytohormone contents are the means of three biological replicates. UP, unpollinated; P, pollinated; CPPU, treatment with $N$-(2-chloro-4-pyridyl)- $N^{\prime}$-phenylurea alone; CPPU + PAC, treatment with $N$-(2-chloro-4-pyridyl)- $N^{\prime}$-phenylurea supplemented with paclobutrazol. Significant differences among treatments as determined by one-way ANOVA $(P<0.05)$ are indicated using different lowercase letters. Error bars represent the standard deviation of the mean $(S D ; n=3)$

(a)
B: CPPU vs UP
C: CPPU+PAC vs UP

A: P vs UP (b)

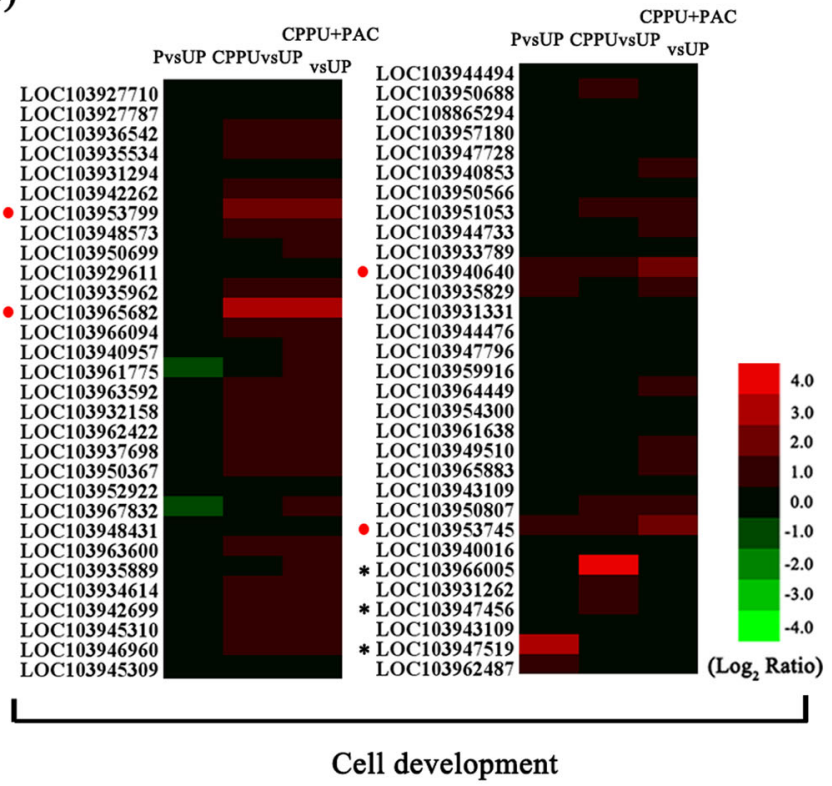

Fig. 4 Distribution of differentially expressed genes (DEGs). a Distribution of total DEGs in pollinated (P) vs. unpollinated (UP), N-(2-chloro-4-pyridyl)$N^{\prime}$-phenylurea (CPPU) vs UP, and CPPU supplemented with paclobutrazol (CPPU + PAC) vs. UP treatment groups. b Relative expression of cell development-related genes. Circles indicate genes discussed in the text, and asterisks denote genes with FPKM $<1$

PAC-supplemented CPPU treatment groups (Fig. 4b; Supplementary Table S3). Compared with the CPPUalone and PAC-supplemented CPPU treatment groups, few cell development-related DEGs were found in the pollinated group, namely, one cyclin, three expansins, and three XTHs were upregulated (Fig. 4b; Supplementary Table S3). These results were consistent with our histological observations. 


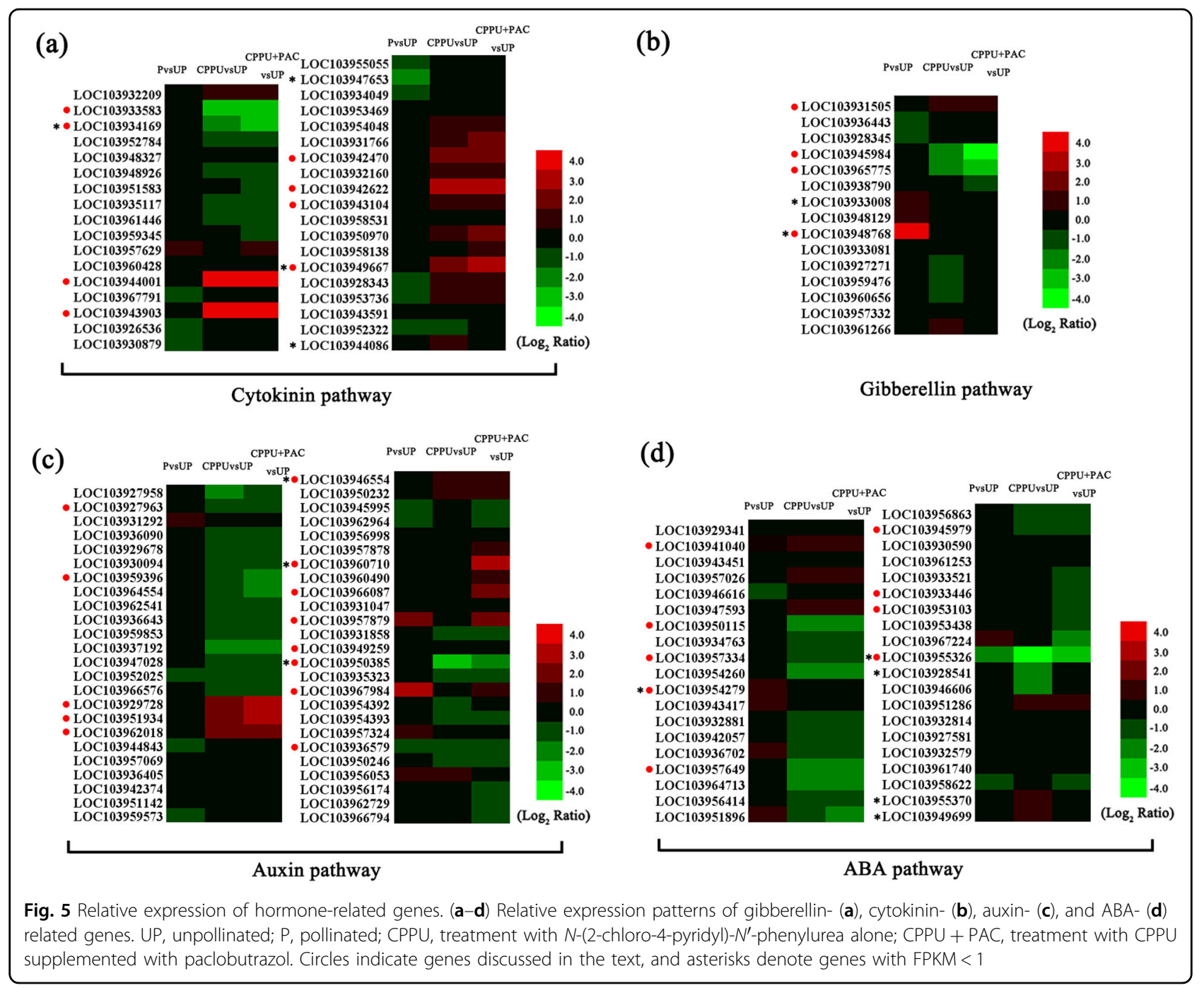

\section{DEGs involved in hormone pathways}

The cytokinin biosynthetic genes adenylate isopentenyltransferase (IPT) and cytokinin riboside 5'monophosphate phosphoribohydrolase (LOG) were significantly downregulated after the CPPU treatment. In particular, PbIPT5-like (LOC03934169) was downregulated three-fold, while PbLOG5 (LOC103933583) was downregulated nearly five-fold (Fig. 5a; Supplementary Table S4). The cytokinin degradation gene Pbcytokinin dehydrogenase 3-like (LOC103944001) was upregulated more than 1000-fold in the CPPU-alone and PAC-supplemented CPPU treatment groups (Fig. 5a; Supplementary Table S4). The expression of genes involved in the cytokinin signaling pathway was also altered. PbAHK4 (LOC103943104), PbAHK5-like (LOC103949667), PbAHP5-like (LOC103943903), PbRR9-like (LOC103942622), and PbRR10-like (LOC103942470) were significantly upregulated by treatment with CPPU alone and CPPU supplemented with PAC (Fig. 5a; Supplementary Table S4). GA20ox and GA3ox are two key genes in the gibberellin biosynthesis pathway. No GA20ox or GA3ox transcripts were found among the DEGs (Fig. 5b, Supplementary Table S5). These results indicate that CPPU treatment does not induce gibberellin biosynthesis at 4 DAA. PbGA2ox8 (LOC103948768) was significantly upregulated more than 70-fold in the pollinated group (Fig. 5b; Supplementary Table S5). We also found two GA2ox genes (LOC103945984 and LOC103965775) and one GASA gene (LOC103931505) that were downregulated and upregulated two-fold, respectively, after both CPPU treatments (Fig. 5b; Supplementary Table S5).

The expression of genes involved in the auxin pathway was altered after the CPPU-alone and PAC-supplemented CPPU treatments. YUCCA is a key enzyme in the auxin biosynthesis pathway. According to the RNA sequencing results, only PbYUCCA4 (LOC103962018) was upregulated five-fold in both the CPPU-alone and PAC-supplemented 
CPPU treatment groups (Fig. 5c; Supplementary Table S6). In addition, two transcripts (LOC103960710 and LOC103966087) were significantly upregulated in the PAC-supplemented CPPU treatment groups (Fig. 5c; Supplementary Table S6). After treatment with CPPU and PAC-supplemented CPPU, three auxin-responsive genes -Pbindole-3-acetic acid-amido synthetase GH3.6 (LOC103946554), Pbauxin binding protein 19a (LOC103951934) and Pbauxin binding protein 20-like (LOC103929728)-were significantly upregulated more than three-fold, while one auxin-responsive genePbindole-3-acetic acid-amido synthetase GH3.17-like (LOC103950385)—was significantly downregulated more than three-fold (Fig. 5c; Supplementary Table S6). Moreover, two auxin-induced genes (LOC103957879 and LOC103967984) were significantly upregulated in the pollinated and PAC-supplemented CPPU treatment groups (Fig. 5c; Supplementary Table S6). In addition, the expression of many ARF and IAA family genes, such as PbIAA11-like (LOC103927963), PbARF3-like (LOC103936579), PbARF9 (LOC103949259), and PbARF19-like (LOC103959396) (Fig. 5c; Supplementary Table S6), was altered in the pollinated, CPPU-alone, and PAC-supplemented CPPU treatment groups.

According to the RNA sequencing results, three 9-cisepoxycarotenoid dioxygenases (NCEDs) (LOC103945979, LOC103955326, and LOC103957334) were downregulated in the CPPU-alone and PAC-supplemented CPPU treatment groups (Fig. 5d; Supplementary Table S7). Among these genes, PbNCED6 (LOC103955326) was downregulated nearly 20-fold after CPPU treatment (Fig. 5d; Supplementary Table S7). Abscisic acid $8^{\prime}$-hydroxylase is involved in the oxidative degradation of abscisic acid. One transcript (LOC103941040) was upregulated in the pollinated, CPPU-alone, and PAC-supplemented CPPU treatment groups (Fig. 5d; Supplementary Table S7); in addition, one transcript (LOC103954279) was upregulated in the pollinated group, while two transcripts (LOC103950115 and LOC103957649) were downregulated in both the CPPU-alone and PAC-supplemented CPPU treatment groups (Fig. 5d; Supplementary Table S7). Furthermore, eight protein phosphatase 2Cs (PP2Cs) were downregulated and nine $P P 2 C s$ were upregulated after the CPPU-alone and PAC-supplemented CPPU treatments (Fig. 5d; Supplementary Table S7). Moreover, some PP2Cs were upregulated in the pollinated group and downregulated in the PAC-supplemented CPPU treatment group, such as PbPP2C23 (LOC103933446) and PbPP2C49 (LOC103953103) (Fig. 5d; Supplementary Table S7).

\section{PbRR9 promotion of PbYUCCA4 expression and suppression of PbNCED6 expression}

Two-component response regulators (RRs) are important transcription factors in the cytokinin signaling pathway. YUCCA and NCED are key enzymes in the auxin biosynthetic pathway and the ABA biosynthesis pathway, respectively. According to the RNA sequencing results, PbRR9 (LOC103942622) and PbNCED6 (LOC103955326) were the most significantly expressed DEGs in the RR and NCED families (Fig. 5b, d; Supplementary Tables S4, S7). The FPKM of PbRR9 increased from 4 to 50, while that of PbNCED6 delined from 1 to 0.1 (Fig. S2; Supplementary Table S9). In addition, the only YUCCA gene upregulated in both CPPU treatments was YUCCAA4 (LOC103962018) (Fig. 5c; Supplementary Table S6). PbRR9, PbYUCCA4, and PbNCED6 were therefore selected to further evaluate the mechanism of CPPU-induced parthenocarpy.

According to the qRT-PCR results, PbRR9 and PbYUCCA4 were significantly upregulated and PbNCED6 was significantly downregulated in the CPPU-alone and PAC-supplemented CPPU treatment groups at 4 DAA (Supplementary Fig. S2). The results of a yeast one-hybrid assay indicated that PbRR9 can combine with the PbYUCCA4 and PbNCED6 promoters (Fig. 6a). Moreover, dual luciferase assays revealed that $P b R R 9$ can promote the activity of the PbYUCCA4 promoter and repress the activity of the PbNCED6 promoter (Fig. 6b, c). To further verify the function of $P b R R 9$, we transiently overexpressed PbRR9 in pear fruits by injection. Successful infection of the 'Dangshansu' fruits was confirmed by monitoring GUS signals (Supplementary Fig. S3). qRTPCR revealed that PbRR9 overexpression significantly upregulated PbYUCCA4 and downregulated PbNCED6 (Fig. 6d-f). These results demonstrate that $P b R R 9$ is upstream of PbYUCCA4 and PbNCED6 and can upregulate PbYUCCA4 and downregulate PbNCED6.

\section{Discussion}

Cytokinin can induce parthenocarpy in many horticultural plants, including watermelon ${ }^{29}$, tomato $^{30}$, grape $^{31}$, and cucumber ${ }^{32}$. Although previous research has shown that CPPU can induce parthenocarpy in pear $^{23}$, the mechanism of induction has been unclear. Parthenocarpy is closely related to the coordinated action of different hormones, among which gibberellin is recognized as a key hormone $e^{5,24,34}$. In tomato, CPPU-induced parthenocarpy is partly dependent on enhanced gibberellin and auxin biosynthesis and the application of PAC can significantly decrease gibberellin content ${ }^{30}$. Our previous studies have indicated that $\mathrm{GA}_{4+7}, 2,4$-dichlorophenoxyacetic acid (2,4-D), and melatonin (MT) can all induce parthenocarpy, which in pear is dependent on gibberellin ${ }^{12,24,35}$. To investigate whether gibberellin also plays a key role in CPPU-induced parthenocarpy in pear, we sprayed CPPU supplemented with PAC (a gibberellin synthesis inhibitor) on bagged 'Dangshansu' pear flowers. We observed no difference in the fruit set rate between the CPPU-alone 


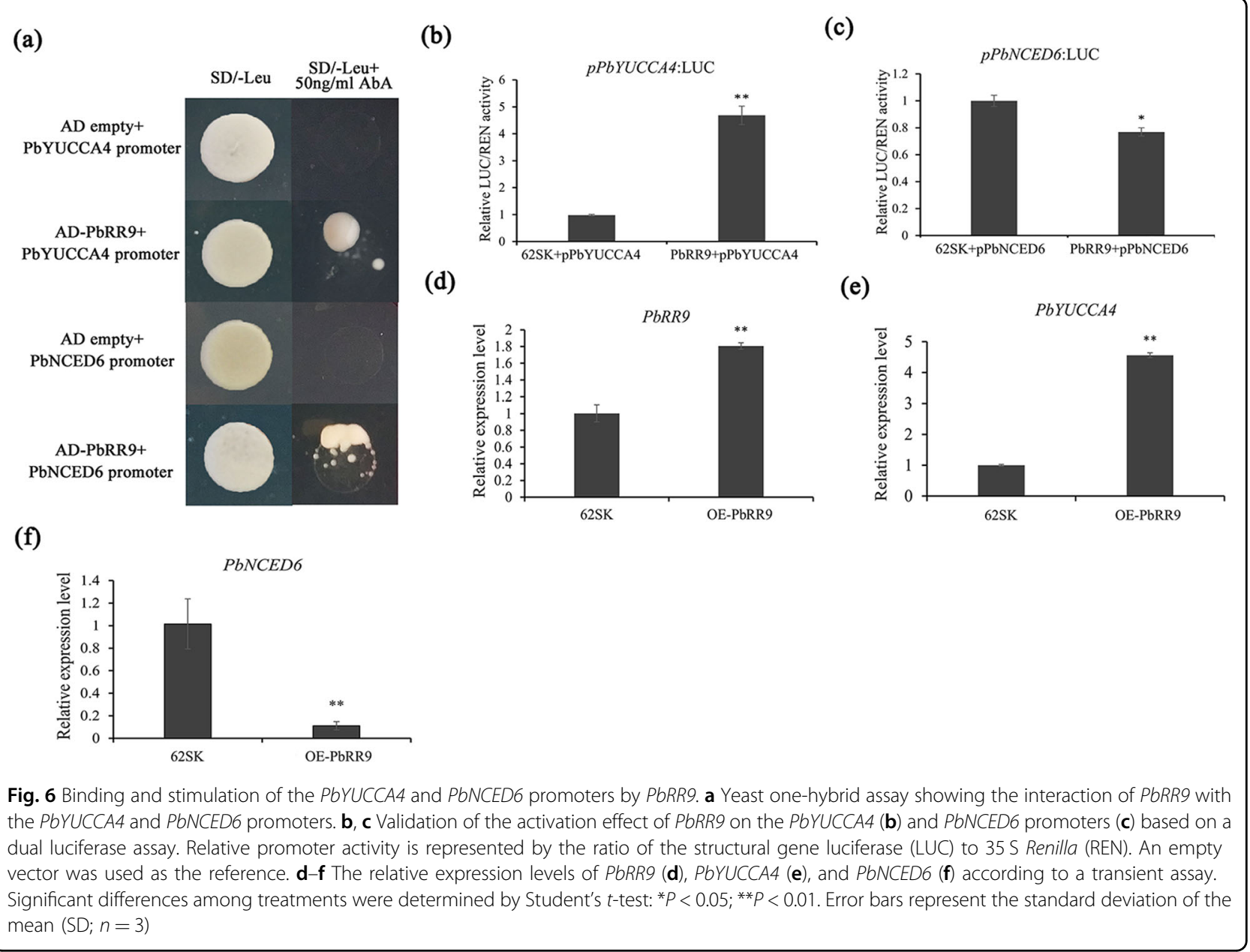

and PAC-supplemented CPPU treatment groups. This result indicates that blocking the biosynthesis of gibberellin does not inhibit CPPU-induced parthenocarpy in 'Dangshansu' pear. We thus speculate that CPPU induces a gibberellin-independent parthenocarpy that is different from that triggered by $\mathrm{GA}_{4+7}, 2,4-\mathrm{D}$ or MT.

After pollination and fertilization, a series of cell division, expansion, and differentiation activities occurs in plants that causes fruit set and development ${ }^{36,37}$. In our study, we found that CPPU can promote cell division and expansion that significantly increases hypanthium thickness and observed that PAC application had no influence on the effect of CPPU on fruit cell development. No differences in hypanthium thickness, cell area, or the number of cell layers were detected between the CPPU-alone and PAC-supplemented CPPU treatment groups. These results suggest that gibberellin does not have an important role in CPPU-induced cell development. The expression of $C D K$ and $C Y C$, two major gene families involved in cell division, is regulated by hormones ${ }^{38,39}$. EXP and XTH regulate cell size and shape by changing cell wall plasticity ${ }^{40,41}$, while cytokinin affects the expression of cell cycle-related genes and cellular processes to initiate parthenocarpic fruit development ${ }^{42,43}$. According to our RNA sequencing results, several CYCs, $C D K s$, and EXPs from different subfamilies were upregulated after CPPU-alone and PAC-supplemented CPPU treatment. These results are consistent with histological observations of cell development, which indicates that CPPU promotes cell division and expansion and that the effect of CPPU is not dependent on gibberellin.

Previous research has suggested that $\mathrm{GA}_{3}$ and $\mathrm{GA}_{4}$ are the major bioactive gibberellins in pear ${ }^{12,24}$. In our study, a phytohormone content assay revealed that neither $\mathrm{GA}_{3}$ nor $\mathrm{GA}_{4}$ content increased after CPPU treatment, and a decrease even was observed in some cases. Moreover, the RNA sequencing analysis uncovered no GA20ox or GA3ox genes that were differentially expressed. GA20ox and GA3ox are key enzymes in the gibberellin biosynthesis pathway, and a change in their expression patterns can influence the level of bioactive gibberellins ${ }^{25}$. Thus, CPPU did not promote the synthesis of GA in CPPU-induced parthenocarpy. The above results support our hypothesis that CPPU-induced parthenocarpy in 'Dangshansu' pear is 
gibberellin independent. In addition, we identified two GA2ox genes and one GASA gene that were downregulated in both CPPU treatment groups. These results may be due to different developmental processes induced by CPPU or to the different tissue localization of different genes ${ }^{44}$.

In addition to our analysis of gibberellins, we found that IAA content was significantly upregulated and ABA content was significantly downregulated after CPPU treatment. Our previous study indicated that a high IAA level and a low ABA level play certain roles in pear parthenocarpy ${ }^{24}$. According to our RNA sequencing results, several genes related to auxin biosynthesis and signaling pathways were differentially expressed after CPPU treatment. YUCCA is a key gene in the auxin biosynthesis pathway, and the upregulation of PbYUCCA4 is consistent with the results of our phytohormone determination. Many genes participating in the auxin signaling pathway, such as SlARF7, SmARF8, and SIIAA9, are related to parthenocarpy ${ }^{16-18,45}$. According to the results of RNA sequencing, PbARF7 (LOC103929678) was significantly downregulated and PbIAA9 (LOC103936405) was significantly upregulated in both CPPU treatments. Auxin may thus play an essential role in CPPU-induced parthenocarpy in 'Dangshansu' pear. ABA is an important hormone that regulates plant growth and development. The ovaries of parthenocarpic mandarin oranges have low $\mathrm{ABA}$ content ${ }^{21}$, which suggests that $\mathrm{ABA}$ plays a negative role in fruit set ${ }^{46}$. In our study, three NCEDs were significantly downregulated, and several $P P 2 C s$ were differentially expressed. NCED is a key enzyme in the ABA synthesis pathway ${ }^{47,48}$, and the downregulation of NCEDs is consistent with the low ABA levels after the CPPU treatment. $P P 2 C s$ are regulators of the ABA signaling pathway ${ }^{49,50}$, and the differential expression of $P P 2 C s$ after CPPU treatment indicates that the ABA pathway is activated by CPPU. ABA may thus also be involved in the process of CPPU-induced parthenocarpy.

To more fully elucidate the mechanism of CPPUinduced parthenocarpy in 'Dangshansu' pear, we further analyzed PbRR9, PbYUCCA4, and PbNCED6. Similar to bacterial two-component phosphorelay signal transduction, the cytokinin signaling transduction model has three components: a hybrid HK receptor that contains both histidine kinase and receiver domains, authentic histidine phosphotransferases (AHPS) and separate response regulators $(R R s)^{51-53}$. RRs play an important role in the cytokinin signaling pathway and regulate the expression of some downstream cytokinin-response genes ${ }^{53,54}$. The results of a yeast one-hybrid assay revealed that $P b R R 9$ can combine with the PbYUCCA4 and PbNCED6 promoters. In addition, dual luciferase assays indicated that PbRR9 promotes the activity of the PbYUCCA4 promoter and represses that of the PbNCED6 promoter. Moreover, we found that PbYUCCA4 was significantly upregulated and $P b N C E D 6$ was significantly downregulated after transient overexpression of $P b R R 9$ in fruits. $P b R R 9$ is an A-type response regulator gene. A-type response regulators are generally regarded as negative regulators of cytokinin signaling ${ }^{53}$; however, a few A-type response regulators have been found to function as positive regulators $^{55-57}$. Furthermore, the expression of YUCCA and NCED can affect the endogenous IAA and ABA contents $^{21,58,59}$. The data presented here therefore indicate that PbRR9 is upstream of PbYUCCA4 and PbNCED6 and that $P b R R 9$ can upregulate $P b Y U C C A 4$ and downregulate $P b N C E D 6$ to increase IAA content and decrease ABA content.

Finally, we observed the undeveloped seeds in mature CPPU-induced parthenocarpic fruits. The presence of these seeds suggests that a signal required for the promotion of seed development might be provided by CPPU.

In summary, our findings increase our understanding of the novel mechanism of CPPU-induced parthenocarpy (Fig. 7). CPPU may initially upregulate $P b R R 9$ and then increase IAA content and decrease ABA content by upregulating PbYUCCA4 and downregulating PbNCED6. Cell division and expansion are subsequently activated and eventually induce parthenocarpy in 'Dangshansu' pear (Fig. 7). Because fertilization may not have been complete in the pollinated group at 4 DAA, we were unable to compare the differences between normal fruiting and parthenocarpy. In addition, 'botanical' and 'accessory' fruit structures are different; consequently, the tissues used in this study are different from those in a related study of tomato, which may have led to different conclusions about the mechanisms of CPPU-induced parthenocarpy. The conclusions of our study are therefore still preliminary.

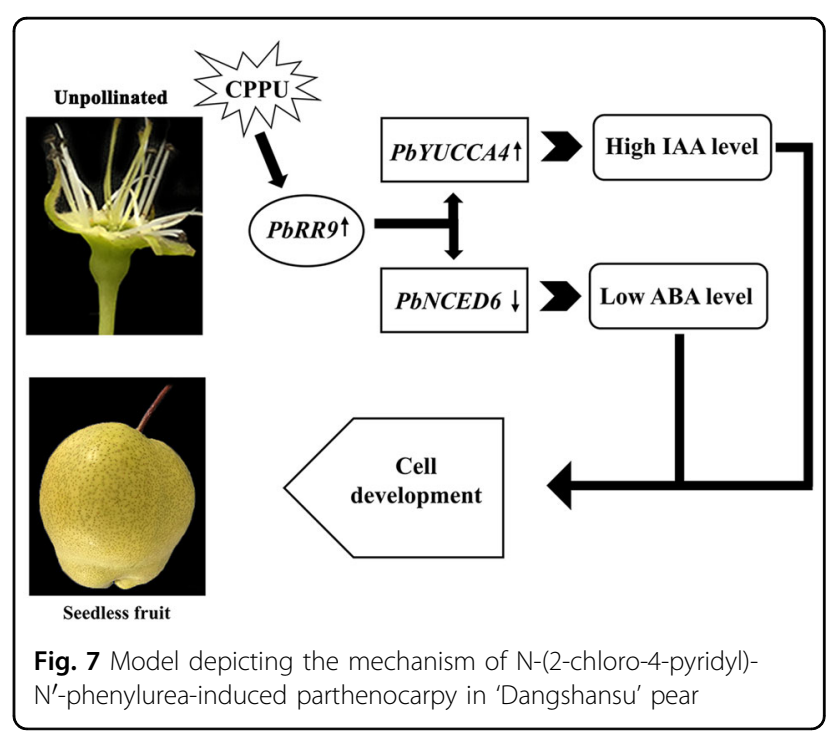




\section{Materials and methods}

Plant materials and experimental treatments

The experimental plants used in this study were 13-year-old 'Dangshansu' pear (Pyrus bretschneideri Rehd.) trees in an orchard located in Qianxian, Shaanxi Province, China $\left(34.25^{\circ} \mathrm{N}, 108.12^{\circ} \mathrm{E} ; 514 \mathrm{~m}\right.$ above sea level). To prevent pollination, we placed bags over the flowers 2 days before anthesis. Four treatments were applied: no pollination (water); pollination; $20 \mathrm{mgl}^{-1}$ CPPU (in water); and $20 \mathrm{mg} \mathrm{l}^{-1} \mathrm{CPPU}$ supplemented with $5 \mu \mathrm{mol} \mathrm{l}^{-1}$ PAC (in water). Flowers in each treatment group were sprayed at anthesis. Fruits were collected at 4, 10, and 152 days after anthesis (DAA).

\section{Determination of fruit set rate}

When all the flowers in the unpollinated group had fallen off (approximately 20 DAA), we removed the bags and calculated the fruit set rate. A total of 120 blooms on three branches were selected to determine the fruit set rate, which was calculated as follows: fruit set rate $(\%)=$ (number of fruits remaining/the total number of fruits) $\times 100 \%$

\section{Determination of fruit quality}

Ten mature fruits of each group were used to measure fruit quality. The total soluble solids content was determined using a portable system (PAL-1 pocket refractometer, Atago, Japan). Fruit firmness was determined on three opposite sides of the surface of each fruit (equatorial region) using a GY-4 texture analyzer (TOP Instrument, China). A Vernier caliper was used to measure the core transverse diameter. The formula used to calculate the fruit shape index was as follows: fruit shape index $=$ fruit suture diameter/polar diameter.

\section{Paraffin sectioning}

Fruits collected at 4 DAA from unpollinated, pollinated, CPPU, and PAC-supplemented CPPU treatment groups were used as samples (Fig. S4). These samples were immediately fixed in formaldehyde-acetic acid-alcohol fixative ${ }^{60}$ and stored at $4{ }^{\circ} \mathrm{C}$. The fruits were dehydrated in an ethanol/xylene series and embedded in paraffin, sectioned into $8-\mu \mathrm{m}$-thick slices, dried, and stained with safranin and fast green. A microscopic imaging system (BX51 + PD72 + IX71, Olympus, Japan) was used to observe the anatomical images. Cell area and tissue thickness were measured using ImageJ software, with three sections from three fruits used for each measurement.

\section{Phytohormone analysis}

Fruits collected at 4 DAA were used as the samples for phytohormone analysis (Supplementary Fig. S4). The fruits $(0.5 \mathrm{~g})$ were ground, and $4 \mathrm{ml}$ of extraction buffer (citric acid, 2,6-di-tert-butyl-4-methylphenol, and methyl alcohol) was added to the fruit samples. After shaking overnight at
$4{ }^{\circ} \mathrm{C}$, the suspension was centrifuged at $10,000 \mathrm{~g}$ for $15 \mathrm{~min}$, and the supernatant was withdrawn. Next, $3 \mathrm{ml}$ of extraction buffer was added to the precipitate, which was shaken at $4{ }^{\circ} \mathrm{C}$ for $1 \mathrm{~h}$ and centrifuged at $10,000 \mathrm{~g}$ for $15 \mathrm{~min}$. The supernatant was then dried under nitrogen gas, dissolved in $300 \mu \mathrm{l}$ methanol $(0.1 \%$ methane acid), and passed through a $0.22-\mu \mathrm{m}$ filter membrane. $\mathrm{GA}_{3}, \mathrm{GA}_{4}, \mathrm{IAA}$, and ABA contents were determined by HPLC-MS/MS (AB SCIEX TripleTOF 5600+, US-MA) using an Acquity UPLC HSS T3 column $(1.8 \mu \mathrm{m} ; 2.1 \times 100 \mathrm{~mm}$; Waters, USA). The injection volume was $2 \mu \mathrm{l}$. The MS conditions were as follows: a spray voltage of $4500 \mathrm{~V}$ and air curtain, nebulizer, and auxiliary gas pressures of 15,65 , and $70 \mathrm{psi}$, respectively. The atomizing temperature was $400{ }^{\circ} \mathrm{C}$. Three independent biological replicates were performed.

\section{Transcriptome analysis}

Fruits collected at 4 DAA were used for RNA sequencing (Supplementary Fig. S4). Total RNA was extracted using an RNA Prep Pure Plant kit (Tiangen, Beijing, China). RNA degradation and contamination were monitored on $1 \%$ agarose gels, and RNA purity was checked on a NanoPhotometer spectrophotometer (Implen, CA, USA). RNA was quantified using a Qubit RNA Assay kit on a Qubit 2.0 fluorometer (Life Technologies, CA, USA), and RNA integrity was assessed using an RNA Nano 6000 Assay kit supplied with the Bioanalyzer 2100 system (Agilent Technologies, CA, USA). A total of $1 \mu \mathrm{g}$ RNA per sample was subjected to RNA sequencing on an Illumina HiSeq instrument. Sequencing libraries were generated using a NEBNext Ultra RNA Library Prep kit for Illumina (NEB, USA), with index codes added to attribute sequences to each sample. The clustering of index-coded samples was performed on a cBot cluster generation system using a TruSeq PE cluster kit v3-cBot-HS (Illumina) according to the manufacturer's instructions. After cluster generation, the library preparations were sequenced on an Illumina HiSeq platform, and 125-bp/150-bp paired-end reads were generated. After constructing a reference genome index in Bowtie v2.2.3, paired-end clean reads were aligned to the reference genome using TopHat v2.0.12. The number of reads mapped to each gene was counted in HTSeq v0.6.1. The RNA-sequencing data from unpollinated fruits were used as controls. A false discovery rate $<0.001$ and a $\mid \log 2$ ratio $\mid>1$ were used as thresholds of the significance of DEGs. Genes were annotated using the 'Dangshansuli' database (http://www.ncbi. nlm.nih.gov/genome/?term=pyrus) as a reference. Three independent biological replications were sequenced and analyzed.

\section{Quantitative real-time PCR (qRT-PCR) validation of gene expression levels}

qRT-PCR analysis was performed on a StepOnePlus Real-Time PCR instrument (ABI, US-MA) using TB 
Green Premix Ex Taq II (Tli RNaseH Plus, Takara, Dalian, China). Total RNA was extracted using an RNA Prep Pure Plant kit (Tiangen), and $1 \mu \mathrm{g}$ of total RNA was then reverse-transcribed to cDNA using a PrimeScript RT reagent kit with gDNA Eraser (Takara). The primers used for qRT-PCR are listed in Supplementary Table S8. PbActin7 (LOC103926846) was used as a reference gene. Three biological replicates were performed. The relative expression levels of the tested genes were calculated using the cycle threshold $\left(C_{t}\right) 2^{-\Delta \Delta C}$ method $^{61}$.

\section{Transient assay}

For the overexpression of PbRR9, the full-length PbRR9 (LOC103942622) coding sequence (CDS) was PCR-amplified from 'Dangshansu' cDNA sources and then inserted into a pGreenII 0029 62-SK vector using a ClonExpress One Step Cloning kit (Vazyme, Nanjing, China). The constructed vector was expressed under the control of the CaMV $35 \mathrm{~S}$ promoter. The complete GUS CDS of the pBI121-GUS plasmid was cloned into the multiple cloning sites (MCS) of the pGreenII 0029 62-SK binary vector to form the pGreenII 0029 62-SK-GUS plasmid. The primers used for CDS amplification are listed in Supplementary Table S8. The resulting plasmid was transferred into Agrobacterium tumefaciens strain GV3101 by the heat-shock method, and the Agrobacterium cells were then grown at $28^{\circ} \mathrm{C}$ in $\mathrm{LB}$ solid medium containing appropriate antibiotics (kanamycin and rifampicin). After incubation for $48 \mathrm{~h}$, the Agrobacterium cells were resuspended in infiltration buffer $\left(10 \mathrm{mM} \mathrm{MgCl}_{2}\right.$, $10 \mathrm{mM}$ MES, and $200 \mu \mathrm{M}$ acetosyringone) and shaken for $3-4 \mathrm{~h}$ at room temperature to achieve a final $\mathrm{OD}_{600}$ of 0.8 prior to injection of pear fruits. Twenty-day-old fruits were used for the injection, which was implemented as described in Spolaore et al. ${ }^{62}$ using the injection volume detailed in Zhai et $\mathrm{al}^{63}$. The negative controls were infiltrated with Agrobacterium containing a pGreenII 0029 62-SK empty vector. Treated fruits were harvested 5 days after injection. Three independent biological replicates were performed. The GUS staining method was as described in Fillatti et al. ${ }^{64}$.

\section{Yeast one-hybrid (Y1H) assay}

$\mathrm{Y} 1 \mathrm{H}$ assays were performed according to the manufacturer's instructions using a Matchmaker Gold Yeast One-Hybrid System kit (Clontech, Mountain View, CA, USA). To construct pAbAi-baits, we ligated approximately 500-bp fragments of the PbYUCCA4 (LOC103962018) and PbNCED6 (LOC103955326) promoters into pAbAi. The complete CDS of PbRR9 was separately inserted into a pGADT7 vector to construct prey-AD vectors. The $\mathrm{pAbAi}$-bait vectors were linearized and transformed into Y1HGold separately. Colonies were selected on a plate with selective synthetic dextrose medium (SD) lacking uracil. Confirmation of the correct integration of the plasmids into the genome of Y1HGold was performed by colony PCR analysis (Matchmaker Insert Check PCR Mix 1; Clontech). After determining the minimal inhibitory concentration of aureobasidin A (AbA) for the bait reporter yeast strains, the AD-prey vectors were transformed into the bait yeast strains and selected on an SD/-Leu/AbA plate. All transformations and screenings were performed three times.

\section{Dual luciferase assay}

PbYUCCA4 and PbNCED6 promoters were amplified from 'Dangshansu' genomic DNA using PrimeSTAR Max Premix (Takara) and then cloned into dual-LUC plasmid pGreenII 0800-LUC. The full-length CDS of $P b R R 9$ was cloned into the MCS (BamHI-HindIII) region of a pGreenII 0029 62-SK binary vector ${ }^{65}$.

Each recombinant plasmid and the pSoup helper plasmid were transferred individually into Agrobacterium strain GV3101. Agrobacterium cells containing PbRR9-62SK were separately mixed with PbYUCCA4 promoter-LUC or PbNCED6 promoter-LUC at a 1:1 ratio before infiltration into 4-week-old Nicotiana benthamiana leaves. After injection, the plants were placed in darkness at room temperature for 3 days. The treated leaves were then collected in $1 \times$ phosphate-buffered solution for the dual luciferase assay. The ratio of firefly luciferase to Renilla luciferase enzyme activities was analyzed using a dual-luciferase reporter assay system (Promega, Madison, WI, USA) on a Tecan Infinite M200pro full-wavelength multifunctional enzyme labeling instrument (TECAN, Hombrechtikon, Switzerland). Three independent biological replicates were performed.

\section{Acknowledgements}

We thank Liwen Bianji, Edanz Group China (www.liwenbianji.cn/ac), for editing the English text of a draft of this manuscript. This work was supported by the China Agriculture Research System (CARS-28-45) and the Primary Research and Development Plan of Shaanxi Province (2017NY-029).

\section{Author contributions}

Z.W., L.X. and L.C. designed the experiments. L.C., T.W., H.L., H.W., H.Z., G.Z. and Y.W. performed the experiments and T.W., H.W., H.L. and H.Z. analyzed the data. G.Z., T.W. and L.C. wrote and revised the manuscript. All authors participated in the research and approved the final manuscript.

Conflict of interest

The authors declare that they have no conflict of interest.

Supplementary Information accompanies this paper at (https://doi.org/ 10.1038/s41438-020-0285-5).

Received: 23 August 2019 Revised: 7 February 2020 Accepted: 2 March 2020

Published online: 01 May 2020

\section{References}

1. Gillaspy, G., Ben-David, H. \& Gruissem, W. Fruit-a development perspective. Plant Cell. 5, 1439-1451 (1993).

2. Goetz, M. et al. Expression of aberrant forms of AUXIN RESPONSE FACTOR8 stimulates parthenocarpy in Arabidopsis and tomato. Plant Physiol. 145, 351-366 (2007). 
3. Gustafson, F. G. Parthenocarpy: natural and artificial. Bot. Rev. 8, 599-654 (1942).

4. Ruan, Y. L., Patrick, J. W., Bouzayen, M., Osorio, S. \& Fernie, A. R. Molecular regulation of seed and fruit set. Trends Plant Sci. 17, 656-665 (2012).

5. Serrani, J. C. et al. Inhibition of auxin transport from the ovaries or from the apical shoot induces parthenocarpic fruit-set in tomato mediated by gibberellins. Plant Physiol. 153, 851-862 (2010).

6. Koshioka, M. et al. Analysis of gibberellins in growing fruits of Lycopersicon esculentum after pollination or treatment with 4-chlorophenoxyacetic acid. J. Hort. Sci. 69, 171-179 (1994).

7. Gustafson, F. G. Auxin distribution in fruits and its significance in fruit development. Am. J. Bot. 26, 189-194 (1939).

8. Joldersma, D. \& Liu, Z. The making of virgin fruit: the molecular and genetic basis of parthenocarpy. J. Exp. Bot. 69, 955-962 (2018).

9. Nitsch, J. P. Growth and morphogenesis of the strawberry as related to auxin. Am. J. Bot. 37, 211-215 (1950).

10. Serrani, J. C., Ruiz-Rivero, O., Fos, M. \& Garcia-Martinez, J. L. Auxin-induced fruitset in tomato is mediated in part by gibberellins. Plant J. 56, 922-934 (2008).

11. Zhang, C., Lee, U. \& Tanabe, K. Hormonal regulation of fruit set, parthenogenesis induction and fruit expansion in Japanese pear. Plant Growth Regul. 55, 231-240 (2008).

12. Cong, L. et al. 2,4-D-induced parthenocarpy in pear is mediated by enhancement of $\mathrm{GA}_{4}$. Biosynth. Physiol. Plant. 166, 812-820 (2018).

13. Acciarri, N. et al. Genetically modified parthenocarpic eggplants: improved fruit productivity under both greenhouse and open field cultivation. BMC Biotechnol. 2, 4 (2002).

14. Rotino, G. L., Perri, E., Zottini, M., Sommer, H. \& Spena, A. Genetic engineering of parthenocarpic plants. Nat. Biotechnol. 15, 1398-1401 (1997).

15. Ren, Z. et al. The auxin receptor homologue in Solanum lycopersicum stimulates tomato fruit set and leaf morphogenesis. J. Exp. Bot. 62, 2815-2826 (2011).

16. De Jong, M., Wolters-Arts, M., Feron, R., Mariani, C. \& Vriezen, W. H. The Solanum lycopersicum auxin response factor 7 (SIARF7) regulates auxin signaling during tomato fruit set and development. Plant J. 57, 160-170 (2009b).

17. Wang, H. et al. The tomato Aux/IAA transcription factor IAA9 is involved in fruit development and leaf morphogenesis. Plant Cell. 17, 2676-2692 (2005).

18. Mazzucato, A. et al. A TILLING allele of the tomato Aux/IAA9 gene offers new insights into fruit set mechanisms and perspectives for breeding seedless tomatoes. Mol. Breed. 35, 22 (2015).

19. Watanabe, M., Segawa, H., Murakami, M., Sagawa, S. \& Komori, S. Effects of plant growth regulators on fruit set and fruit shape of parthenocarpic apple fruits. J. Jpn. Soc. Hortic. Sci. 77, 350-357 (2008).

20. Prosser, M. V. \& Jackson, G. A. D. Induction of parthenocarpy in Rosa arvensis huds with gibberellic acid. Nature 184, 108-108 (1959).

21. Mesejo, C., Reig, C., Martínez-Fuentes, A. \& Agustí, M. Parthenocarpic fruit production in loquat (Eriobotrya japonica Lindl.) by using gibberellic acid. Sci. Hortic. 126, 37-41 (2010).

22. Jung, C. J. et al. Transcriptional changes of gibberellin oxidase genes in grapevines with or without gibberellin application during inflorescence development. J. Plant Res. 127, 359-371 (2014).

23. Niu, Q. F. et al. Effects of exogenous application of $\mathrm{GA}_{4+7}$ and N-2-chloro-4pyridyl-N'-phenylurea on induced parthenocarpy and fruit quality in Pyrus pyrifolia 'Cuiguan'. Plant Growth Regul. 76, 251-258 (2015).

24. Liu, L. L. et al. Histological, hormonal and transcriptomic reveal the changes upon gibberellin-induced parthenocarpy in pear fruit. Hortic. Res. 5, 1 (2018).

25. Giacomelli, L. et al. Gibberellin metabolismin Vitis vinifera L. during bloom and fruit-set: functional characterizationand evolution of grapevine gibberellin oxidases. J. Exp. Bot. 64, 4403-4419 (2013).

26. García-Hurtado, N. et al. The characterization of transgenic tomato overexpressing gibberellin 20-oxidase reveals induction of parthenocarpic fruit growth, higher yield, and alteration of the gibberellin biosynthetic pathway. J. Exp. Bot. 63, 5803-5813 (2012).

27. Martinez-Bello, L., Moritz, T. \& Lopez-Diaz, I. Silencing C19-GA 2-oxidases induces parthenocarpic development and inhibits lateral branching in tomato plants. J. Exp. Bot. 66, 5897-5910 (2015).

28. Martí, C. et al. Silencing of DELLA induces facultative parthenocarpy in tomato fruits. Plant J. 52, 865-876 (2007).

29. Hayata, Y., Niimi, Y. \& Iwasaki, N. Synthetic cytokinin-1-(2=chloro=4=pyridy)3-phenylurea (CPPU)-promotes fruit set and induces parthenocarpy in watermelon. J. Am. Soc. Hortic. Sci. 120, 997-1000 (1995).
30. Ding, J. et al. Cytokinin induced parthenocarpic fruit development in tomato is partly dependent on enhanced gibberellin and auxin biosynthesis. PLOS ONE 8, e70080 (2013).

31. Lu, L. et al. Auxin- and cytokinin-induced berries set in grapevine partly rely on enhanced gibberellin biosynthesis. Tree Genet. Genomes 12, 41 (2016).

32. Qian, C. et al. Effects of exogenous application of CPPU, NAA and $\mathrm{GA}_{4+7}$ on parthenocarpy and fruit quality in cucumber (Cucumis sativus L.). Food Chem. 243, 410-413 (2017).

33. Wu, J. et al. The genome of the pear (Pyrus bretschneideri Rehd.). Genome Res. 23, 396-408 (2013)

34. Tang, N., Deng, W., Hu, G., Hu, N. \& Li, Z. Transcriptome profiling reveals the regulatory mechanism underlying pollination dependent and parthenocarpic fruit set mainly mediated by auxin and gibberellin. PLOS ONE 10, e0125355 (2015).

35. Liu, J. L. et al. Melatonin induces parthenocarpy by regulating genes in gibberellin pathways of 'Starkrimson' pear (Pyrus communis L.). Front Plant Sci. 9, 946 (2018).

36. Vivian-Smith, A. \& Koltunow, A. M. Genetic analysis of growth-regulatorinduced parthenocarpy in Arabidopsis. Plant Physiol. 121, 437-452 (1999).

37. Seymour, G. B. \& Granell, A. Fruit development and ripening. Annu. Rev. Plant Biol. 64, 219-241 (2013).

38. Inzé, D. \& Veylder, L. D. Cell cycle regulation in plant development. Annu. Rev. Genet. 40, 77-105 (2006).

39. Mesejo, C. et al. Gibberellin reactivates and maintains ovary-wall cell division causing fruit set in parthenocarpic Citrus species. Plant Sci. 247, 13-24 (2016).

40. Marowa, P., Ding, A. \& Kong, Y. Expansins: roles in plant growth and potential applications in crop improvement. Plant Cell Rep. 35, 949-965 (2016).

41. Atkinson, R. G., Johnston, S. L., Yauk, Y., Sharma, N. N. \& Schröder, R. Analysis of xyloglucan endotransglucosylase/hydrolase (XTH) gene families in kiwifruit and apple. Postharvest Biol. Tec. 51, 149-157 (2009).

42. Fu, F. Q., Mao, W. H., Shi, K., Zhou, Y. H. \& Yu, J. Q. Spatio-temporal changes in cell division, endoreduplication and expression of cell cycle-related genes in pollinated and plant growth substances-treated ovaries of cucumber. Plant Biol. 12, 98-107 (2010)

43. Matsuo, S., Kikuchi, K., Fukuda, M., Honda, I. \& Imanishi, S. Roles and regulation of cytokinins in tomato fruit development. J. Exp. Bot. 63, 5569-5579 (2012).

44. Li, C. et al. Comprehensive expression analysis of Arabidopsis GA2-oxidase genes and their functional insights. Plant Sci. 285, 1-13 (2019).

45. Du, L. et al. SmARF8, a transcription factor involved in parthenocarpy in eggplant. Mol. Genet. Genomes 291, 93-105 (2016).

46. Vriezen, W. H., Feron, R., Maretto, F., Keijman, J. \& Mariani, C. Changes in tomato ovary transcriptome demonstrate complex hormonal regulation of fruit set. $N$. Phytol. 177, 60-76 (2008).

47. Luchi, S. et al. Regulation of drought tolerance by gene manipulation of 9cisepoxycarotenoid dioxygenase, a key enzyme in abscisic acid biosynthesis in Arabidopsis. Plant J. 27, 325-333 (2001).

48. Lefebvre, V. et al. Functional analysis of Arabidopsis NCED6 and NCED9 genes indicates that $\mathrm{ABA}$ synthesized in the endosperm is involved in the induction of seed dormancy. Plant J. 45, 309-319 (2006).

49. Sheen, J. Mutational analysis of protein phosphatase $2 \mathrm{C}$ involved in abscisic acid signal transduction in higher plants. Proc. Natl. Acad. Sci. USA 95, 975-980 (1998).

50. Han, S. et al. Modulation of aba signaling by altering vxg $\Phi$ motif of pp2cs in, oryza sativa. Mol. Plant 10, 1190-1205 (2017).

51. Higuchi, M. et al. In planta functions of the Arabidopsis cytokinin receptor family. Proc. Natl. Acad. Sci. USA 101, 8821-8826 (2004).

52. Schaller, G. E., Shiu, S. H. \& Armitage, J. P. Two-component systems and their co-option for eukaryotic signal transduction. Curr. Biol. 21, R320-R330 (2011).

53. Kieber, J. J. \& Schaller, G. E. Cytokinin signaling in plant development. Development 145, dev149344 (2018).

54. Zubo, Y. et al. Cytokinin induces genome-wide binding of the type-B response regulator ARR10 to regulate growth and development in Arabidopsis. Proc. Natl. Acad. Sci. USA 114, E5995-E6004 (2017).

55. Osakabe, Y. et al. Overexpression of Arabidopsis response regulators, ARR4/ ATRR1/IBC7 and ARR8/ATRR3, alters cytokinin responses differentially in the shoot and in callus formation. Biochem. Biophys. Res. Commun. 293, 806-815 (2002). 
56. Leibfried, A. et al. WUSCHEL controls meristem function by direct regulation of cytokinin-inducible response regulators. Nature 438, 1172-1175 (2005).

57. Lee, B. H. et al. Studies of aberrant phyllotaxy1 mutants of maize indicate complex interactions between auxin and cytokinin signaling in the shoot apical meristem. Plant Physiol. 150, 205-216 (2009).

58. Ji, K. et al. Non-climacteric ripening in strawberry fruit is linked to $A B A$, FaNCED2 and FaCYP707A1. Funct. Plant Biol. 39, 351-357 (2012).

59. Wang, Y., Ding, G., Gu, T., Ding, J. \& Li, Y. Bioinformatic and expression analyses on carotenoid dioxygenase genes in fruit development and abiotic stress responses in Fragaria vesca. Mol. Genet. Genomics 292, 895-907 (2017).

60. Henwood, A. What is the best procedure to remove formalin pigment from formalin pigment from formaldehyde-acetic acid-alcohol fixed tissues? J. Histotechnol. 33, 109-111 (2010).
61. Livak, K. J. \& Schmittgen, T. D. Analysis of relative gene expression data using real-time quantitative $P C R$ and the $2^{-\Delta \Delta C t}$ method. Methods 25, 402-408 (2001).

62. Spolaore, S., Trainotti, L. \& Casadoro, G. A simple protocol for transient gene expression in ripe fleshy fruit mediated by Agrobacterium. J. Exp. Bot. 52, 845-850 (2001).

63. Zhai, R. et al. Two MYB transcription factors regulate flavonoid biosynthesis in pear fruit (Pyrus bretschneideri Rehd.). J. Exp. Bot. 67, 1275-1284 (2016).

64. Fillatti, J. J., Kiser, J., Rose, R. \& Comai, L. Efficient transfer of a glyphosate tolerance gene into tomato using a binary agrobacterium tumefaciens vector. Nat. Biotechnol. 5, 726-730 (1987).

65. Hellens, R. P. et al. Transient expression vectors for functional genomics, quantification of promoter activity and RNA silencing in plants. Plant Methods 1, 13 (2005). 